\title{
Clipping of ipsilateral posterior communicating and superior cerebellar artery aneurysms
}

\author{
Babu G. Welch, M.D.
}

Departments of Neurosurgery and Radiology, The University of Texas Southwestern Medical Center, Dallas, Texas

The case is a 55-year-old female who presented with dizziness as the chief complaint. She has a family history of two relatives with subarachnoid hemorrhage. Digital subtraction angiography revealed the presence of a left-sided posterior communicating artery aneurysm and an ipsilateral superior cerebellar artery (SCA) aneurysm. Due to the smaller nature of the SCA, a decision was made to proceed with surgical clipping of both lesions through a pterional approach. A narrated video with illustrations depicts the intraoperative management of these lesions with postoperative angiography results.

The video can be found here: http://youtu.be/HCHToSsXv-4.

KEY WORDS aneurysm; posterior communicating artery; superior cerebellar artery; microsurgery 